\title{
On the Benefits of Probabilistic Constellation Shaping in Flex-Grid/MCF Dynamic Optical Backbone Networks
}

\author{
Jordi Perelló \\ Universitat Politècnica de Catalunya \\ (UPC) - BarcelonaTech \\ Barcelona, Spain \\ perello@ac.upc.edu
}

\author{
Joan M. Gené \\ Universitat Politècnica de Catalunya \\ (UPC) - BarcelonaTech \\ Barcelona, Spain \\ joan.gene@upc.edu
}

\author{
Salvatore Spadaro \\ Universitat Politècnica de Catalunya \\ (UPC) - BarcelonaTech \\ Barcelona, Spain \\ salvatore.spadaro@upc.edu
}

\begin{abstract}
In this paper, we propose a novel methodology for estimating the worst-case spectral efficiency $(\mathrm{b} / \mathrm{s} / \mathrm{Hz})$ of end-to-end paths across a Multi-Core Fiber (MCF)-enabled Flex-Grid optical network, accounting for physical link and intra-node noise, together with the inter-core crosstalk impairment appearing during the transmission of optical signals over MCFs. In particular, we employ the proposed methodology to quantify the benefits of Probabilistic Constellation Shaping (PCS) versus traditional polarization-multiplexed modulation formats in two reference backbone networks. From the obtained results, PCS yields admissible offered load gains close to $13 \%$ under $1 \%$ bandwidth blocking probability.
\end{abstract}

Keywords-Flex-Grid, SDM, MCFs, spectral efficiency, probabilistic constellation shaping.

\section{INTRODUCTION}

Elastic Optical Networks (EONs) implementing Flex-Grid and Spatial Division Multiplexing (SDM) technologies [1] have been identified as key candidates to realize future optical network infrastructures, given their superior flexibility and capacity, far beyond the non-linear Shannon limit of standard Single Mode Fibers (SMFs). In support of SDM, high corecount Multi-Core Fibers (MCFs) with very low inter-core crosstalk (XT) values have already been designed and fabricated (e.g., the homogeneous 22-core or heterogeneous 30-core MCFs reported in [2] and [3]), paving the way to next-generation SDM-enabled Flex-Grid/MCF optical networks.

In order to optimize the spectral usage in Flex-Grid optical networks, the so-called distance-adaptive Modulation Format (MF) selection is usually performed. In this strategy, the most efficient yet feasible MF is chosen for supporting any new lightpath, taking into account its maximum transmission reach and end-to-end transmission distance from lightpath's source to destination. Most works addressing pure Flex-Grid (over standard SMFs) and Flex-Grid/SDM network optimization issues contemplate the selection of traditional PolarizationMultiplexed (PM) MFs, ranging from PM-BPSK up to advanced PM- $m$-QAM ones (e.g., as in [4][5]).

Besides, Probabilistic Constellation Shaping (PCS) has recently received considerable research attention, providing a fine-grained software-defined trade-off between achievable Spectral Efficiency (SE) and transmission distance [6]. The wide tunability of PCS has been demonstrated across the full distance range, from Data Center Interconnects (DCI) to trans-Pacific, using a single 32-GBaud hardware platform [7].
This work aims to evaluate the benefits that PCS can yield in Flex-Grid/MCF dynamic optical backbone networks. For this purpose, we propose a novel worst-case methodology to estimate the attainable SE when using PCS along end-to-end paths across a Flex-Grid/MCF optical network. Employing this methodology, we then compare the performance of PCS versus traditional MFs in two reference backbone network scenarios, a national and a continental-wide one.

The remainder of the paper continues as follows. Section II presents the proposed path worst-case SE estimation methodology. Section III describes the Route, Modulation format, Core and Spectrum Assignment (RMCSA) heuristic that we employ to allocate spectral super-channels in the conducted experiments. Section IV presents the obtained results. Finally, Section V concludes the paper.

\section{PATH SPECTRAL EFFICIENCY CONSTRAINTS}

The maximum $\mathrm{SE}(\mathrm{b} / \mathrm{s} / \mathrm{Hz})$ per polarization-multiplexed spatial path of total distance $L$ using an adaptive modulation such as PCS and ideal Nyquist pulse shaping is given by [8]:

$$
S E_{\max }=2 \cdot \log _{2}\left(1+\frac{1}{3\left[\chi P_{A S E}^{2} / 4\right]^{1 / 3}+\kappa \cdot L}\right)
$$

where $P_{A S E}$ is the Amplified Spontaneous Emission (ASE) power within the signal channel's bandwidth.

We consider transparent lumped amplification with $85-\mathrm{km}$ spans and an attenuation parameter $\alpha=0.2 \mathrm{~dB} / \mathrm{km}$. We also consider $10-\mathrm{dB}$ extra losses for the signals going through every intermediate node and the corresponding optical amplifiers to compensate for them. In all cases, a 5-dB optical amplifier Noise Figure (NF) is assumed. Nonlinear Interference Noise (NLIN) is quantified by parameter $\chi$ [9]. To calculate it, a nonlinear parameter $\gamma=1.3 W^{-1} \mathrm{~km}^{-1}$ is assumed, together with a dispersion parameter $D=17 \mathrm{ps} \cdot \mathrm{nm}^{-1} \mathrm{~km}^{-1}$. Also, a fully loaded $\mathrm{C}$-band is considered all the time, which corresponds to the maximum (i.e., worst-case) NLIN level.

Finally, $\kappa$ denotes the aggregate $X T$ per unit distance experienced by a core from signals co-propagating at the same wavelength in all other cores simultaneously. When all cores are active, the maximum level of XT is generated, which again corresponds to the worst-case scenario. We assume an optimized MCF layout providing the same $\kappa$ for all cores. As shown in [10], there exists an optimum XT level of about $-55 \mathrm{~dB} / \mathrm{km}$ that maximizes the aggregate capacity. This corresponds to an optimum number of cores for each MCF outer diameter. This 
value is taken as a reference in our calculations assuming the MCF used has been optimized. Under such assumption, any number of cores can be taken.

We should remark that the model used to estimate the nonlinear parameter $\chi$ is based on the well-known GaussianNoise Model [9]. As mentioned before, ideal Nyquist pulse shaping is assumed, which is seen as realistic given current stateof-the art technology. Provided that all spans are considered uniform, the calculation of the end-to-end $\chi$ is essentially the nonlinear parameter of a single span $\chi_{s}$ multiplied by the number of whole spans in the path (follow [9] for details). The last span in every internodal link needs to be treated separately, as its length becomes a fraction of a regular span and the gain of the corresponding amplifier should be reduced accordingly. The following formula is reached eventually:

$$
\chi=\chi_{s}\left(N_{s}+\sum_{i=1}^{N_{l}}\left\{1-G_{s}^{-\rho_{i}}\right\}^{2}\right)
$$

where $N_{s}$ stands for the number of whole spans and $G_{s}$ refers to the gain of the whole-span amplifiers. The number of links in the path is $N_{l}$ and $0 \leq \rho_{i} \leq 1$ refers to the span fraction to complete the i-th link.

Fractional spans require special treatment when calculating $P_{A S E}$ as well. Also, because nodes are assumed to be lossy, the corresponding amplifiers need to be included. The overall expression reads:

$$
P_{A S E}=h \cdot f\left\{\left(G_{s} N_{s}+\sum_{i=1}^{N_{l}} G_{s}^{\rho_{i}}\right)+N_{n} G_{n}\right\} F \cdot R_{s}
$$

where $h$ is Planck's constant, $f$ is the optical frequency, $F$ is the amplifier's noise figure and $R_{s}$ is the symbol rate. $G_{n}$ refers to the gain of node amplifiers and $N_{n}$ to the number of intermediate nodes in the path. Notice that the product $\chi P_{A S E}^{2}$ is symbol rate independent, which makes $S E_{\max }$ to be symbol rate independent as well.

The suggested procedure provides the worst-case $S E_{\text {max }}$ calculation. This value can pre-computed only once (i.e., offline), thus simplifying resource management. The busier the network it gets the more accurate the calculations become. In other words, the spectrum assignment becomes more efficient when spectral efficiency is most required.

\section{RMCSA HEURISTIC}

To decide on the route, modulation format, core and spectrum portion to be assigned to spectral super-channels carrying the requested demands, we employ a fast lightweight RMCSA heuristic, which receives a pre-computed set of $K$ candidate shortest physical paths (in $\mathrm{km}$ ) between each pair of source-destination nodes in the network. For each candidate path, its SE is pre-computed using the proposed methodology in Section II, as the attainable SE along it when using PCS. Alternatively, when using traditional MFs, the most efficient MF offering a SE lower or equal than $S E_{\max }$ resulting from eq. (1) is selected, and its SE will be the one of the candidate path. For example, when eq. (1) applied to a candidate path gives an $S E_{\max }$ value equal to $9.25 \mathrm{~b} / \mathrm{s} / \mathrm{Hz}$, this will be the $\mathrm{SE}$ attainable along it when using PCS. Conversely, when traditional MFs are used, we consider PM-BPSK, PM-QPSK, PM-16-QAM, PM64-QAM and PM-256-QAM. So, in this path PM-16-QAM will be used, with $\mathrm{SE}=8 \mathrm{~b} / \mathrm{s} / \mathrm{Hz}$.
When an incoming demand request of $B(\mathrm{~Gb} / \mathrm{s})$ arrives at the network, the RMCSA heuristic starts exploring its possible allocation over a spectral super-channel, along any of the precomputed candidate paths between its source and destination nodes, from the shortest to the longest one, on a first-fit fashion. For each path, the number of required Frequency Slots (FS) is computed as $n_{f S}=\lceil(B / S E+G B) / W\rceil$, where $S E$ is the precomputed spectral efficiency of the path, $G B$ is the guard-band width between adjacent spectral super-channels (in $\mathrm{GHz}$ ) and $W$ is the FS spectral width (also in GHz). Note that no guard-bands are considered when calculating $S E_{\max }$ in the previously proposed worst-case methodology in Section II. Nonetheless, we do account for them here as an additional safety margin. Next, an available spectral portion of $n_{f s}$ adjacent FSs is sought in any core along the candidate path, starting with the one with the lowest index on, also on a first fit fashion. If such spectrum portion is found, the request is considered served using these resources. Otherwise, if no spectrum portion is found along any core of any candidate path from its source to destination node, the demand request is considered as blocked.

The proposed RMCSA heuristic enforces core continuity end-to-end. This decision has been motivated by the potential deployment of cost-effective SDM-Reconfigurable Optical Add \& Drop Multiplexers (ROADMs) without lane-change support, which deliver similar performance to fully-flexible Independent Switching (InS) ones in dynamic Flex-Grid/MCF networks [5].

\section{NUMERICAL RESULTS}

To assess the benefits of PCS versus traditional MFs in dynamic Flex-Grid/MCF optical networks, an ad-hoc Pythonbased simulator has been used. Two reference backbone networks have been considered for the study, namely, the national Deutsche Telekom network DT12 (12 nodes, 20 bidirectional links, avg. link length $243 \mathrm{~km}$ ) and the PanEuropean EON16 (16 nodes, 23 bidirectional links, avg. link length $486 \mathrm{~km}$ ), whose details can be found in [5]. We assume both networks equipped with SDM-ROADMs without lane change support and 22-core MCF links, which corresponds to a well-proven MCF core-count technology. Moreover, we assume that the entire $4 \mathrm{THz} \mathrm{C}$-Band is made available in all MCF cores, discretized into $320 \mathrm{FSs}$ of $12.5 \mathrm{GHz}$ width.

In order to start illustrating the potential benefits of PCS against traditional MFs, Fig. 1 depicts the average path SE obtained when pre-computing the end-to-end candidate paths in DT12 and EON16 networks offline (setting $K=1,3$ and 6). As seen, PCS can increase average path SE from $2.02(K=1)$ to 2.56 $\mathrm{b} / \mathrm{s} / \mathrm{Hz}(K=6)$ in the DT12 network, compared to using traditional MFs, representing relative increments from 17 up to $28 \%$. In the EON16 network, path SE values are generally lower, given the longer distances between source-destination node pairs. Nonetheless, PCS can still raise average path SE from $1.34(K=6)$ up to $2.05 \mathrm{~b} / \mathrm{s} / \mathrm{Hz}(K=1)$, i.e., showing relative increments from 17 up to $23 \%$.

It is noteworthy from Fig. 1 that average path SE gains follow inverse trends in DT12 and EON16 networks. While SE gains of using PCS in the DT12 seem higher in longer candidate paths, in the EON16 the opposite occurs. The reason behind this behavior is the following. Increasing the $K$ value in the DT12 results in the computation of a number of paths whose SE falls slightly under $12 \mathrm{~b} / \mathrm{s} / \mathrm{Hz}$. Therefore, when traditional MFs are 
used, PM-16-QAM must be employed, reducing the SE of such paths down to $8 \mathrm{~b} / \mathrm{s} / \mathrm{Hz}$, which introduces very noticeable average SE differences against PCS, particularly when $K=6$. In the EON16, higher $K$ values also lead to the computation of longer paths. However, while their SE generally reduces, in many cases it stays close but still above $8 \mathrm{~b} / \mathrm{s} / \mathrm{Hz}$. This allows using PM-16-QAM, resulting in similar SE as with PCS.

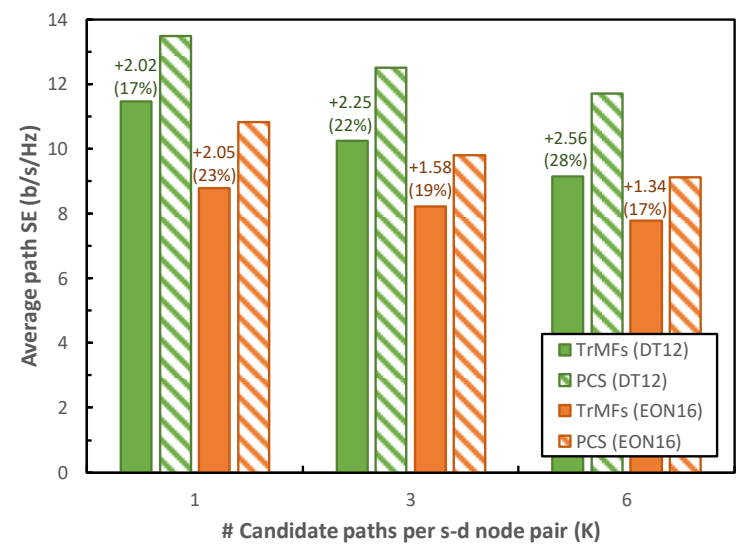

Fig. 1. Average pre-computed path SE (b/s/Hz) in DT12 and EON16 networks $(K=1,3$ and 6$)$ when using traditional MFs (TrMFs) or PCS.

Furthermore, we have conducted additional experiments to also evaluate the average Bandwidth Blocking Probability (BBP) versus offered load in DT12 and EON16 networks, when using either traditional MFs or PCS. In these experiments, demand requests arrive at the network following a Poisson traffic process, describing exponentially distributed inter-arrival and holding times, with mean values equal to IAT and HT, respectively. To generate different loads, we fix IAT $=1.0$ and scale HT accordingly (offered load $=$ HT/IAT). When a new demand request arrives, we consider it of 400,800 or $1200 \mathrm{~Gb} / \mathrm{s}$ with probability $0.4,0.4$ or 0.2 , respectively. Incoming demand requests should be allocated over spectral super-channels, adding $10 \mathrm{GHz}$ guard-bands between adjacent ones. Moreover, an intermediate $K=3$ value has been set in the RMCSA heuristic. The obtained results are shown in Fig. 2, where 150x103 demand requests are offered per execution.

Looking at Fig. 2, PCS significantly reduces BBP in DT12 and EON16 networks when compared to using traditional MFs, especially as the offered load to the network increases. Identifying $\mathrm{BBP}=1 \%$ as an acceptable network operational scenario, PCS allows increasing the offered load by 1100 and 1000 in DT12 and EON16 networks, respectively. These absolute values represent promising offered load increments of almost $13 \%$ in both cases, which should pay the reasonable Digital Signal Processing (DSP) complexity required to realize PCS [6]. We should point out, however, that the observed admissible network load gains under $\mathrm{BBP}=1 \%$ remain below the average path SE gains that PCS can potentially achieve, previously illustrated in Fig. 1. We believe that such reduction can in part be caused by the additional spectrum fragmentation that can appear in dynamic Flex-Grid/MCF scenarios when using PCS, given the wider range of spectrum allocation options in terms of required number of FSs. To counteract these effects, the application of more advanced RMCSA heuristics, able to minimize or even totally avoid fragmentation in the network may be beneficial, which is left for future work.

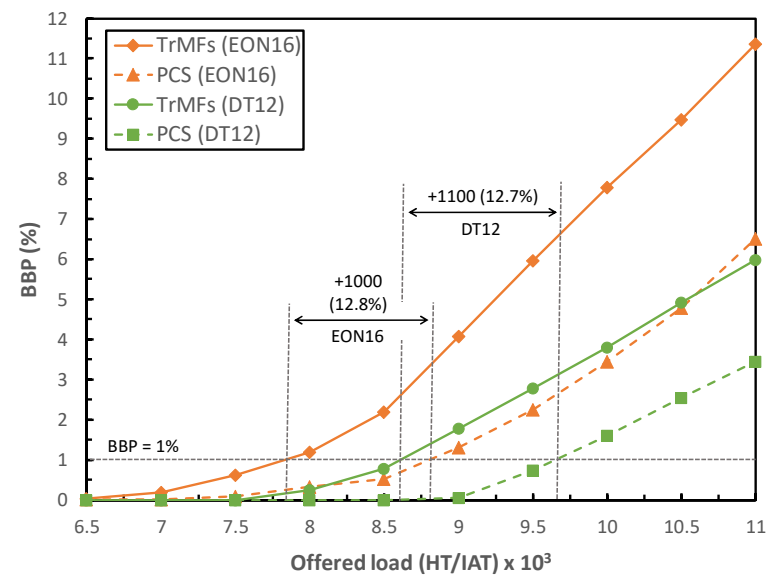

Fig. 2. Average BBP vs. offered load in DT12 and EON16 networks when using traditional MFs (TrMFs) or PCS.

\section{CONCLUSIONS}

This paper evaluates the benefits of PCS in Flex-Grid/MCF dynamic optical backbone networks. From the obtained results, PCS can increase the admissible offered load up to almost $13 \%$ under $\mathrm{BBP}=1 \%$. This makes PCS a very interesting modulation option in this kind of networks, whose benefits may be further increased by using fragmentation-aware RMCSA heuristics. Future work should also investigate the implementation of PCS in experimental Flex-Grid/MCF network test-beds, e.g., using commercially available PCS-enabled DSPs at the moment.

\section{ACKNOWLEDGEMENTS}

This work has been supported by the Spanish Government through project ALLIANCE (TEC2017-90034-C2-1-R and TEC2017-90034-C2-2-R) with FEDER contribution. Moreover, it has been supported by the Spanish Thematic Network under contract RED2018-102585-T (Go2Edge).

\section{REFERENCES}

[1] D. Klonidis, et al., "Spectrally and spatially flexible optical network planning and operations," IEEE Commun. Mag., vol. 53, no. 2, pp. 6978, Feb. 2015.

[2] B.J. Puttnam, et al., " $2.15 \mathrm{~Pb} / \mathrm{s}$ transmission using a 22 core homogeneous single-mode multi-core fiber and wideband optical comb," Proc. ECOC 2015, Valencia (Spain), Sept. 2015.

[3] Y. Amma, et al., "High-density multicore fiber with heterogeneous core arrangement," Proc. OFC 2015, Los Angeles, CA, Mar. 2015.

[4] R. Goscien, K. Walkowiak, M. Klinkowski, "Distance-Adaptive Transmission in Cloud-Ready Elastic Optical Networks," IEEE/OSA J. Opt. Commun. Netw., vol. 6, no. 10, pp. 816-828, Oct. 2014.

[5] R. Rumipamba-Zambrano, et al., "Space continuity constraint in dynamic Flex-Grid/SDM optical core networks: An evaluation with spatial and spectral super-channels," Computer Communications, vol. 126, pp. 3849, Aug. 2018

[6] J. Cho and P.J. Winzer, "Probabilistic Constellation Shaping for Optical Fiber Communications," IEEE/OSA J. Light. Technol., vol. 37, no. 6, pp. 1590-1607, Mar. 2019.

[7] J.M. Gené, et al., "Experimental Demonstration of Widely Tunable Rate/Reach Adaptation from $80 \mathrm{~km}$ to $12,000 \mathrm{~km}$ using Probabilistic Constellation Shaping," Proc. OFC 2020, San Diego, CA, Mar. 2020.

[8] T. Hayashi, et al., "Uncoupled multi-core fiber enhancing signal-to-noise ratio," Opt. Express, vol. 20, no. 26, p. B94, Dec. 2012.

[9] P. Poggiolini et al. "The GN-model of fiber non-linear propagation and its applications," IEEE/OSA J. Light. Technol., vol. 32, no. 4, pp. 694 721, Feb. 2014.

[10] J.M. Gené, et al., "Towards broadly optimum multi-core fiber designs," Proc. ECOC 2019, Dublin, Ireland, 2019. 